\title{
Conhecimento Simbólico na Álgebra da Lógica de Venn
}

\author{
Bruno Ramos MendonçA \\ Universidade Federal de Santa Maria
}

\begin{abstract}
This paper reconstructs Venn's algebraic logic and identifies some of the philosophical notions concerning the nature of symbolic knowledge underlying his work. We show that Venn, in facing philosophical problems associated with his algebraic logic, needs to articulate the symbolic knowledge notions of ecthetic function and of surrogative function. The paper explains those notions based on the systematization of the functions of symbolic knowledge that we find in the recent philosophical literature. This paper also situates Venn's work within the 19th Century efforts towards a symbolization of logic, and, through an analysis of a historical case, clarifies the notions of ecthetic function and of surrogative function.
\end{abstract}

Keywords: Symbolic knowledge; algebra of logic; syllogistic; Venn diagrams.

\section{Introdução}

Nesse trabalho reconstruímos o programa de lógica simbólica de John Venn em Symbolic Logic $(1881 ; 1894)$. Venn, lógico mais conhecido pela criação do sistema de diagramas que leva seu nome, é importante representante da tradição da álgebra da lógica do século XIX. Essa tradição constitui-se num marco entre os primeiros esforços sistemáticos de desenvolvimento da lógica simbólica. Nosso trabalho colabora com investigação em curso sobre a natureza do conhecimento simbólico. Por "conhecimento simbólico" entendemos conhecimento obtido a partir do mero trato com símbolos tais como sentenças, equações, fórmulas etc. A essa investigação interessa analisar que funções permitem que os símbolos cumpram um papel epistemológico. Dado que o conhecimento simbólico cumpre papel fundamental no desenvolvimento das ciências formais, a investigação sobre o conhecimento simbólico por vezes articula-se na pesquisa historiográfica sobre as origens modernas da lógica e da matemática. Assim, o trabalho que aqui propomos justifica-se na medida em que reconstruir o programa de lógica simbólica de Venn permite verificar como as funções do conhecimento simbólico estão em jogo numa proposta pioneira de simbolização da lógica.

Verificaremos que Venn articula noções sobre conhecimento simbólico para solucionar duas questões filosóficas associadas a sua álgebra da lógica. Venn interessa-se pela questão sobre a relação entre sua álgebra da lógica e silogística, e pelo problema da relação entre lógica e matemática. Consideremos a primeira questão mencionada. A silogística ${ }^{1}$ é a lógica dos silogismos, argumentos cujo par de premissas

Principia 16(3): 471-488 (2012).

Published by NEL — Epistemology and Logic Research Group, Federal University of Santa Catarina (UFSC), Brazil. 
e conclusão são proposições categóricas. Nessas formas argumentativas há uma "triangulação" de informações, i.e., inferimos que relação dois termos mantêm entre si a partir da relação que eles mantêm com um terceiro, o termo médio. Abaixo segue um exemplo de silogismo:

Alguns homens são filósofos

Todos os filósofos são lógicos

Logo, alguns homens são lógicos

Já em Aristóteles apresenta-se teoria completa e correta da silogística, mas apenas com essa teoria não podemos tratar uma série de outras formas argumentativas. Logo interessou aos lógicos do século XIX desenvolver sistemas mais potentes. Nesse sentido, verificaremos que a álgebra da lógica de Venn oferece tratamento não apenas aos silogismos, mas também a diversas outras formas argumentativas, formadas ou não por proposições categóricas. Surge, contudo, a seguinte questão: que relação mantém-se entre esse sistema mais potente de lógica e a silogística?

A relação entre lógica e matemática apresenta-se como um problema para Venn porque simbolizar algebricamente a lógica levanta a questão sobre se essa disciplina é ou não parte da matemática. Uma opinião generalizada, em meados do século XIX, é a de que as duas disciplinas formais são precisamente diferenciáveis, opinião por vezes associada a conclusões negativas sobre os projetos de álgebra da lógica. Assim, a álgebra da lógica de Venn foi alvo de críticas por supostamente sustentar falsidades e atribuir significado lógico a símbolos algébricos que não o possuem de fato. Logo a questão sobre a relação entre lógica e matemática apresenta-se a Venn como o problema de diferenciar precisamente uma interpretação matemática de seu sistema de álgebra simbólica de uma interpretação lógica. Esse artigo está dividido nas seguintes seções. Na primeira seção, apresentamos a álgebra da lógica de Venn. Já na segunda seção, apresentamos a resposta de Venn aos problemas filosóficos associados a sua álgebra da lógica, e, assim, mostramos como Venn articula noções sobre conhecimento simbólico ao tentar responder aquelas questões filosóficas.

\section{2. Álgebra da Lógica de Venn}

Em Symbolic Logic (1894), Venn propõe uma álgebra da lógica. A tradição de álgebra da lógica, que se desenvolve sistematicamente a partir do século XIX caracteriza-se por aplicar o simbolismo algébrico na formalização lógica. Em termos gerais, Venn formaliza proposições como equações e formaliza deduções como solução de conjuntos de equações. Logo, essa lógica simbólica reduz toda proposição à seguinte forma básica:

$$
\alpha=\beta
$$

Principia 16(3): 471-488 (2012). 
Nessa fórmula, podemos substituir $\alpha$ e $\beta$ por uma gama de termos não lógicos $(a, b, c, \ldots x, y, z \ldots)$, em especial, podemos substituí-los pelos valores 0 e 1 . Além disso, podemos substituir $\alpha$ e $\beta$ por termos complexos formados pela aplicação de operações lógicas a serem apresentadas abaixo. Numa interpretação predicativa, $\alpha$ e $\beta$ representam classes, i.e., coleções de coisas (Venn 1894, p.35). Em especial, os valores 0 e 1 representam, respectivamente, os conjuntos vazio e universo (Venn 1894, p.63). Numa interpretação proposicional, por outro lado, $\alpha$ e $\beta$ representam proposições. Nesse caso, o valor 1 representa o "verdadeiro" e o valor 0 representa o "falso" (Venn 1894, p.436-9). ${ }^{2}$ De acordo com Venn (1894, p.250-2), a interpretação de 1 , seja em sentido predicativo, seja em sentido proposicional depende sempre de considerações contextuais. Assim, 1 significa um universo de extensão variável: em certos contextos, o universo do discurso pode ser, por exemplo, o conjunto de todos os homens existentes, enquanto em outros pode ser um conjunto mais restrito, ou mais amplo, ou incomparável.

Segundo Venn, em equações lógicas a relação de identidade não tem o mesmo significado que em equações matemáticas. Em matemática, essa relação significa "identidade [...] com respeito a alguma característica apenas" (Venn 1894, p.70. Nossa tradução). ${ }^{3}$ Em lógica, por outro lado, identidade é identidade absoluta. Logo podemos remeter a diferença que Venn traça entre identidade matemática e identidade lógica à distinção, bastante usual em metafísica, entre as noções de "identidade qualitativa" e "identidade numérica". 4

$\mathrm{Na}$ álgebra da lógica de Venn, podemos representar as proposições categóricas universais com as seguintes equações:

$$
\begin{array}{ll}
a \cdot b=0 & \text { (Nenhum A é B) } \\
\text { não- } a \cdot b=0 & \text { (Todo B é A) } \\
a \cdot \text { não- } b=0 & \text { (Todo A é B) } \\
\text { não- } a \cdot \text { não- } b=0 & \text { (Tudo não-A é B) }
\end{array}
$$

A forma proposicional "Todo A é B" é simbolizada como "Não existe A e não-B". Notemos, portanto, como a álgebra da lógica de Venn descarta a pressuposição existencial das proposições universais (Venn 1894, p.159). Ora, essa é a razão pela qual a álgebra da lógica de Venn não satisfaz uma interpretação intensional: dado que é possível que classes sejam vazias, de informações sobre suas notas características nem sempre é possível inferir informações sobre suas extensões (Grattan-Guinness 2000, p.61). Por outro lado, notemos que a álgebra da lógica de Venn concebe as operações lógicas como operações sobre termos que geram termos complexos (Venn 1894, p.33). Dessa forma, entende-se a negação como operação sobre termos, o que permite a construção de formas proposicionais não previstas na silogística, tais como a quarta na lista. A álgebra da lógica de Venn prevê as operações lógicas de conjunção e de disjunção que são simbolizadas, respectivamente, com os símbolos algébricos 
de multiplicação “" e soma "+". Além disso, a álgebra da lógica de Venn prevê as operações de subtração lógica e de divisão lógica que são representadas, respectivamente, pelos símbolos algébricos de subtração "-" e de divisão "/". A negação de termo "não-A", por sua vez, Venn não a concebe como operação independente, mas sim enquanto aplicação especial da operação de subtração lógica. Na álgebra da lógica de Venn, "não-A" é igual a "1 1 A", i.e., a extensão do universo do discurso exceto A (Venn 1894, p.64). Por conseguinte, tal como o universo de discurso, a extensão de "não-A" varia contextualmente. Assim, Venn (1894, p.249-50) descarta a tese tradicional de que termos negativos possuem extensão infinita.

Podemos também simbolizar aquelas formas proposicionais através das seguintes equações:

$$
\begin{array}{ll}
(a \cdot \text { não- } b)+(\text { não- } a \cdot b)+(\text { não- } a \cdot \text { não- } b)=1 & (\text { Nenhum A é B) } \\
(\text { não- } a \cdot \text { não- } b)+(a \cdot \text { não- } b)+(a \cdot b)=1 & (\text { Todo B é A) } \\
(\text { não- } a \cdot \text { não- } b)+(\text { não- } a \cdot b)+(a \cdot b)=1 & \text { (Todo A é B) } \\
(\text { não- } a \cdot b)+(a \cdot \text { não- } b)+(a \cdot b)=1 & \text { (Tudo é A ou B) }
\end{array}
$$

Por fim, na álgebra da lógica de Venn, podemos formalizar proposições universais com uso de "0/0", i.e., da divisão lógica da classe vazia por si mesma. Na álgebra da lógica de Venn "0/0" significa uma classe com extensão indefinida. Assim, por exemplo, podemos formalizar uma proposição universal afirmativa através da equação " $a=0 / 0 \cdot b$ ", o que significa "A é idêntico a uma porção indefinida da extensão de B”. Na álgebra da lógica do século XIX, a representação de proposições particulares apresenta-se como problema importante. A solução oferecida por Boole foi representar uma proposição da forma "Algum A é B" através da seguinte equação:

$$
a \cdot b=u
$$

Nessa equação, “ $u$ " representa uma classe de extensão parcialmente indefinida cuja característica distintiva é possuir ao menos um membro (Grattan-Guinness 2000, p.41-2). No entanto tal formalização enfrenta dificuldades. Numa proposição da forma "Algum A é B" afirma-se que ao menos um A é B, mas não se informa quantos são nem quem são esses As. Ora, a formalização de Boole encontra dificuldades justamente em preservar esse aspecto parcialmente indeterminado da quantificação existencial (Geach 1980, p.57). Desse modo, a formalização acaba reconhecendo como válidos argumentos, tais como o seguinte, claramente inválidos:

$$
\begin{aligned}
& a=u \\
& b=u \\
& a=b
\end{aligned}
$$

Principia 16(3): 471-488 (2012). 
Diante dessa dificuldade da formalização booleana, poderíamos concluir com Kneale (1948, p.165) que não existe algo como a classe " $u$ " cuja característica distintiva é possuir ao menos um único membro. Essa é a conclusão a que chega Venn na segunda edição de Symbolic Logic (1894). Se na primeira edição de seu livro Venn adota a formalização booleana, em 1894 Venn prefere formalizar proposições particulares com recurso ao símbolo de desigualdade ">" (Venn 1894, p.184-5). Assim, na álgebra da lógica de Venn uma proposição categórica da forma "Algum A é B" é formalizada do seguinte modo:

$$
a \cdot b>0
$$

A formalização de proposições como equações permite traçar uma correspondência entre propriedades formais de operações lógicas e propriedades formais de operações algébricas. Venn (1894, p.54-70) oferece uma lista de propriedades formais satisfeitas pela álgebra da lógica de Venn:

- Comutatividade da soma lógica: " $a+b=b+a$ ";

- Associatividade da soma lógica: " $a+(b+c)=(a+b)+c$ ";

- Distributividade da soma lógica: " $a+(b \cdot c)=(a+b) \cdot(a+c)$ ";

- Comutatividade da multiplicação lógica: " $a \cdot b=b \cdot c$ ";

- Associatividade da multiplicação lógica: " $a \cdot(b \cdot c)=(a \cdot b) \cdot c$ ";

- Distributividade da multiplicação lógica: " $a \cdot(b+c)=a b+a c$ ";

- Lei da idempotência da multiplicação lógica: " $a n=a$ ", em que " $n$ " é qualquer número natural maior que 0 ;

- " $a-(b-c)=a-b+c$ ";

- Elemento neutro da multiplicação lógica: " $a \cdot 1=a$ ";

- Elemento nulo da multiplicação lógica: " $a \cdot 0=0$ ";

- Lei da não contradição: “ $a \cdot(1-)=0$ ”.

Venn resume nos seguintes termos a forma geral do processo dedutivo associado a sua álgebra da lógica:

Tome as equações dadas e as analise em todos os seus elementos constituintes, a saber, em todas as negações últimas que elas envolvem e que coletivamente formam seu significado. Então tome a função dada, a respeito da qual nos foi pedido que encontre seu valor, e faça a síntese exigida. $\mathrm{Ou}$ seja, construa sucessivamente cada parte dela, empregando para isso as negações mencionadas. Esse último estágio é, de fato, um estágio de rejeição, pois nós começamos pelo desenvolvimento de uma função dada em todo o seu complemento de classes potenciais, e então excluímos tanto quanto se mostrou desaparecer em função da análise prévia. Tendo assim passado pela Análise e pela Síntese sobra um último passo, nomeadamente o passo de eliminação. Pode-se exigir que a função dada seja expressa em termos de

Principia 16(3): 471-488 (2012). 
parte apenas dos termos envolvidos na equação (Venn 1894, p.400. Nossa tradução). ${ }^{5}$

Para ilustrar esse processo, consideremos o seguinte problema: dadas as premissas " $x \cdot y=a$ " e " $y \cdot z=c$ ", encontremos o valor da função " $x \cdot z$ " em função apenas dos termos "a" e "c" (Venn 1894, p.396-7). Ora, de acordo com a passagem acima, o primeiro passo dedutivo consiste em analisar a informação contida nas premissas. Aplicando propriedades algébricas e variadas regras de transformação, podemos derivar das premissas as seguintes equações, nas quais o símbolo “” indica a operação lógica de negação de um termo (Por exemplo, “ $a$ " " significa o termo negativo "não-a"):

$$
\begin{aligned}
& (x \cdot y) \cdot a^{\prime}=0 \\
& \left(x^{\prime} \cdot y \cdot a\right)+\left(x \cdot y^{\prime} \cdot a\right)+\left(x^{\prime} \cdot y^{\prime} \cdot a\right)=0 \\
& (y \cdot z) \cdot c^{\prime}=0 \\
& \left(y^{\prime} \cdot z \cdot c\right)+\left(y \cdot z^{\prime} \cdot c\right)+\left(y^{\prime} \cdot z^{\prime} \cdot c\right)=0
\end{aligned}
$$

Por certa regra de transformação, podemos então analisar essas equações numa lista de "negações últimas":

$$
\begin{aligned}
& x \cdot y \cdot a^{\prime}=0 \\
& x^{\prime} \cdot y \cdot a=0 \\
& x \cdot y^{\prime} \cdot a=0 \\
& x^{\prime} \cdot y^{\prime} \cdot a=0 \\
& y \cdot z \cdot c^{\prime}=0 \\
& y^{\prime} \cdot z \cdot c=0 \\
& y \cdot z^{\prime} \cdot c=0 \\
& y^{\prime} \cdot z^{\prime} \cdot c=0
\end{aligned}
$$

O segundo passo dessa dedução é sintetizar a informação da função pedida. Assim, em primeiro lugar, desenvolvemos toda a informação possível da função " $x \cdot z$ ":

$$
\begin{aligned}
x \cdot z=x \cdot z \cdot[(a \cdot c \cdot y) & +\left(a \cdot c \cdot y^{\prime}\right)+\left(a \cdot c^{\prime} \cdot y\right)+\left(a \cdot c^{\prime} \cdot y^{\prime}\right) \\
& \left.+\left(a^{\prime} \cdot c \cdot y\right)+\left(a^{\prime} \cdot c \cdot y^{\prime}\right)+\left(a^{\prime} \cdot c^{\prime} \cdot y\right)+\left(a^{\prime} \cdot c^{\prime} \cdot y^{\prime}\right)\right]
\end{aligned}
$$

Depois, comparando a informação possível da função com as "negações" contidas nas premissas, rejeitamos uma série de possibilidades. Obtemos então a informação da conclusão em função da informação das premissas:

$$
x \cdot z=x \cdot z \cdot\left[(a \cdot c \cdot y)+\left(a^{\prime} \cdot c^{\prime} \cdot y^{\prime}\right)\right]
$$

Principia 16(3): 471-488 (2012). 
O passo final é a eliminação de informação irrelevante da conclusão. Assim, o problema pedia que a informação de " $x \cdot z$ " fosse apresentada apenas em termos de " $a$ " e "c". Portanto, as ocorrências de outros termos na equação devem ser substituídas por "0/0". Na álgebra da lógica de Venn, como dissemos acima, "0/0" significa uma classe cuja extensão é indefinida ou irrelevante:

$$
x \cdot z=0 / 0 \cdot\left[(a \cdot c \cdot 0 / 0)+\left(a^{\prime} \cdot c^{\prime} \cdot 0 / 0\right)\right]
$$

\section{A solução de Venn e o papel do conhecimento simbólico}

A fim de clarificar a resposta de Venn às questões subjacentes a sua teoria lógica precisamos considerar diferentes funções do conhecimento simbólico. Uma sistematização bastante completa das funções do conhecimento simbólico encontramos em Esquisabel (2012). Em primeiro lugar, Esquisabel mostra que o papel epistemológico fundamental dos símbolos é substituir o conhecimento intuitivo. Em termos gerais, conhecimento intuitivo significa conhecimento por acesso imediato aos objetos estudados, conhecimento que raramente os seres humanos possuem. Portanto o conhecimento simbólico cumpre uma função subrogativa: usamos símbolos para substituir o acesso ao domínio de objetos estudado, de tal modo que o mero trato com os símbolos permite descobrir propriedades satisfeitas por esse domínio de objetos (Esquisabel 2012, p.6). À função subrogativa associam-se outras duas funções epistemológicas. O conhecimento simbólico satisfaz uma função operativa, pois podemos descobrir verdades sobre o domínio estudado por manipulação transformativa do simbolismo. Nesse sentido, o conhecimento simbólico é conhecimento por cálculo. Além disso, o conhecimento simbólico satisfaz uma função ectética, i.e., os símbolos mostram em sua estrutura gráfica propriedades do domínio de objetos representado. Por exemplo, a seguinte equação " $(a+b)+c=a+(b+c)$ ” mostra em sua estrutura gráfica a propriedade de associatividade que o domínio de objetos representado satisfaz. Para além dessas funções epistemológicas, os símbolos podem satisfazer uma função heurística, pois sugerem extensões do domínio de objetos representado, e também podem satisfazer uma função psicotécnica na medida em que tornam a obtenção de conhecimento uma atividade mais simples e eficiente (Esquisabel 2012, p.21-2).

Lassalle Casanave (2012, p.53-5), por sua vez, caracteriza três modos em que o conhecimento simbólico pode satisfazer a função subrogativa. Em primeiro lugar, os símbolos podem subrogar em sentido estrito: nesse caso, entendemos que a cada elemento componente do simbolismo corresponde um elemento do domínio representado. Por outro lado, o conhecimento simbólico pode subrogar em sentido lato. Nesse sentido, embora o simbolismo, como um todo, subrogue um domínio de objetos, determinados componentes do simbolismo não subrogam. Esse modo Lassalle 
Casanave chama de "subrogação como extensão conservativa", pois se entende que os elementos não subrogativos do simbolismo são elimináveis do sistema sem perda de resultados sobre o domínio representado. Tudo que podemos descobrir sobre o domínio de objetos representado com uso de elementos ideais (elimináveis) devemos poder descobrir sem o uso desses elementos. Por fim, Lassalle Casanave prevê que o conhecimento simbólico pode satisfazer a função subrogativa sem representar qualquer domínio de objetos em particular, mas simplesmente estruturas formais que podem ou não estar subjacentes a diferentes domínios de objetos.

Por outro lado, a explicação clássica de como os símbolos satisfazem a função ectética apela à noção de "semelhança". De acordo com essa explicação, há uma relação de semelhança entre simbolismo e domínio de objetos representados. Recorrendo ao exemplo anteriormente apresentado, a equação " $(a+b)+c=a+(b+c)$ " mostraria a propriedade de associatividade devido à semelhança entre essa propriedade formal e a estrutura gráfica da equação, i.e., a ordem de representação dos símbolos seria semelhante a certa ordem na relação entre os objetos. Vale notar, contudo, que essa explicação está longe de constituir consenso, sendo objeto de intenso debate na literatura recente. ${ }^{6} \mathrm{O}$ que, sim, constitui consenso é o caráter estrutural da função ectética, i.e., o conhecimento simbólico satisfaz a função ectética representando uma estrutura formal subjacente ao domínio de objetos representado. Portanto o conhecimento simbólico envolve idealização ${ }^{7}$ do domínio de objetos representado, pois representa apenas algumas de suas propriedades, desconsiderando outras tantas (Esquisabel 2012, p.36).

Articulando o significado das diferentes funções do conhecimento simbólico, Venn responde aquelas questões subjacentes a sua álgebra da lógica, a saber, a questão sobre a relação mantida entre sua álgebra da lógica e a silogística e a questão sobre a relação mantida entre lógica e matemática. Sobre a relação entre sua álgebra da lógica e a silogística, Venn afirma:
[A] Lógica Comum, de fato, não deve ser considerada mais substituída pe- las generalizações do Sistema Simbólico do que é Euclides por aquelas da Geometria Analítica. [...] O sistema mais restrito tem sua vantagem parti- cular devido ao fato de que, sendo por comparação mais concreto, é mais fácil para um iniciante entender; há assim menos risco de que ele falhe ao exercitar a faculdade do pensamento e meramente conduza-se com destreza no uso de uma fórmula; e está muito mais proximamente conectada com as experiências e necessidades práticas da vida ordinária. O sistema mais ge- ral, por outro lado, tem capacidade vastamente estendida, exercita muito mais completamente a faculdade da abstração, e corrige e aumenta as bases científicas do sistema mais restrito (Venn, p.xxvi, xxvii. Nossa tradução). ${ }^{8}$

Na passagem acima, Venn afirma que a álgebra da lógica de Venn é uma generalização da silogística. Isso significa não apenas que ela é mais potente que a silogística, 
mas também que ela oferece tratamento uniforme a todo o domínio estendido de formas argumentativas. Ou seja, a álgebra da lógica de Venn mostraria que a validade tanto de silogismos quanto de argumentos não silogísticos depende de um mesmo conjunto de princípios lógicos. De acordo com Venn, essa generalização é obtida por abstração da lógica silogística levada a cabo no desenvolvimento de sua álgebra da lógica. "Abstrair" significa nesse caso eliminar alguns dos elementos da formalização silogística. O caso mais importante nesse processo é a eliminação da distinção sujeito e predicado. Na teoria silogística, essa distinção cumpre papel fundamental: por exemplo, uma proposição da forma "Todo A é B" é, nessa teoria, formalizada como a combinação do sujeito "A" com o predicado "B". Ora, diversas vezes apontouse a inadequação formal dessa distinção. Assim, Angelelli (1980, p.104-5) critica-a porque ela identifica dois fenômenos lógicos distintos, a saber, a subordinação de uma classe a outra (por exemplo, em "Todo homem é racional") e o pertencimento a uma classe (por exemplo, em "Sócrates é racional"). Em suma, esses críticos argumentam que essa distinção define equivocadamente sujeito e predicado. Mas vale notar que, para esses autores, há uma distinção entre sujeito e predicado logicamente adequada, embora ela não seja a oferecida pela teoria silogística. Venn, por outro lado, descarta qualquer distinção lógica entre sujeito e predicado. De acordo com Venn (1894, p.27), em primeiro lugar, justifica-se a eliminação de tal distinção dada sua irrelevância lógica. Fica claro que falta peso lógico a essa distinção quando consideramos o seguinte exemplo. Tomemos o diagrama de Venn na Figura 1: apenas pela inspeção do diagrama não podemos saber se a forma proposicional representada é "Nenhum A é B" ou "Nenhum B é A", i.e., não nos é exigido especificar o que é sujeito e o que é predicado (Sautter 2010, p.2).

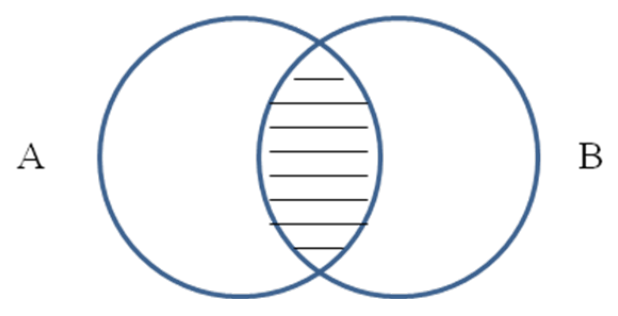

Figura 1

Além disso, Venn aponta uma razão prática para justificar o descarte da distinção sujeito e predicado. Segundo Venn (1876, p.486), embora seja possível atribuir sujeito e predicado a qualquer proposição, o uso dessa distinção encobre o conteúdo informacional das proposições formalizadas. Em suma, na álgebra da lógica de Venn, as proposições têm sua forma lógica reduzida a das proposições téticas, procedimento que, segundo Church (1972), recebeu influência do projeto de Brentano 
de revisão da lógica tradicional. ${ }^{9}$ Ou seja, na álgebra da lógica de Venn, toda proposição é a negação (no caso das proposições universais) ou afirmação (no caso das proposições particulares) da existência de entidades designadas por um dado termo, seja ele simples ou complexo (Venn, 1894, p.24).

Portanto, segundo Venn, a sua álgebra da lógica é o resultado da generalização da silogística obtida pela abstração de certos elementos dessa teoria lógica. ${ }^{10}$ Ora, podemos notar que nesse processo de abstração joga papel central a função ectética do conhecimento simbólico, especificamente no seu caráter de representação estrutural do domínio de objetos estudado. O conhecimento simbólico permite o processo de abstração da silogística porque, ao fixar-se na representação de uma estrutura formal subjacente ao domínio de objetos subrogado, necessariamente descarta a representação de uma série de propriedades desse domínio.

A concepção de Venn da relação entre lógica e matemática é influenciada pelas ideias de Peacock (1791-1858) sobre a natureza da álgebra. Peacock, procurando responder a questão filosófica sobre a natureza dos números não naturais e em especial dos números negativos (Pycior 1981, p.27-31), sustenta uma concepção dupla de álgebra. Por um lado, existiria a álgebra aritmética, i.e., a álgebra cujo simbolismo subroga o domínio dos números naturais. Por outro lado, existiria a álgebra simbólica obtida pela suspensão de regras de operação da álgebra aritmética. Para tornar isso claro, consideremos o seguinte exemplo: a operação de subtração em álgebra aritmética permite apenas que subtraiamos de um dado número outro número menor ou igual a ele, i.e., é possível " $a-b=c$ " se e somente se " $b$ " for menor ou igual a " $a$ ". No entanto, em álgebra simbólica, é possível generalizar a operação de subtração, de modo a tornar possível " $a-b=c$ " mesmo quando o valor de " $b$ " é maior do que o de " $a$ ". Podemos remeter a diferença fundamental entre álgebra simbólica e álgebra aritmética às distintas maneiras de satisfazer a função subrogativa do conhecimento simbólico, i.e., enquanto a álgebra aritmética subroga estritamente o domínio dos números naturais, a álgebra simbólica subroga estruturas formais que podem estar subjacentes a diferentes domínios de discurso (Pycior 1981, p.34-36). Peacock concebe a relação entre essas álgebras nos seguintes termos:

Qualquer forma que seja algebricamente equivalente a outra, quando expressa em símbolos gerais, deve ser verdadeira, seja o que for que esses símbolos denotem.

Reciprocamente, se nós descobrimos uma forma equivalente em álgebra aritmética ou em qualquer outra ciência subordinada, quando os símbolos são gerais em forma mas específicos em sua natureza, o mesmo deve ser uma forma equivalente quando os símbolos são gerais em sua natureza assim como em sua forma (Peacock 1830 apud Pycior 1981, p.38. Nossa tradução). ${ }^{11}$

Primeiramente, precisamos clarificar o uso de certas expressões nessa passagem. 
Na passagem acima, Peacock caracteriza a álgebra simbólica como um sistema simbólico "geral em forma e natureza". A álgebra aritmética, por sua vez, é o exemplo paradigmático de sistema simbólico "geral em forma mas específico em natureza". Ser "geral em forma" significa usar variáveis e parâmetros. Ser "geral em natureza" e ser "específico em natureza" significam, respectivamente, subrogar estruturas formais e subrogar estritamente um domínio de objetos. Com a expressão "formas equivalentes", Peacock denomina equações (Durand-Richard 2000, p.156-7). A passagem acima é analisável em duas teses. Segundo a primeira tese, a álgebra simbólica compõe-se de verdades independentes de qualquer interpretação particular que satisfaçam. De acordo com a segunda tese, a álgebra simbólica preserva toda verdade da álgebra aritmética ou de qualquer outra álgebra estritamente subrogativa.

A concepção de álgebra de Venn é semelhante a de Peacock. Em primeiro lugar, Venn (1894, p.xvii-iii) oferece dupla caracterização à álgebra: por um lado a álgebra seria uma linguagem, e por outro lado ela seria uma ciência interessada especificamente em estudar o domínio das quantidades. Além disso, segundo Venn, a linguagem algébrica pode ser (e constantemente é) aplicada na formalização de diferentes domínios científicos, entre eles o domínio da lógica. Ou seja, Venn também reconhece a existência de uma álgebra subrogadora de estruturas formais e uma variedade de álgebras estritamente subrogativas subordinadas, a la Peacock, àquela álgebra. Com base nesse modo de conceber a álgebra, Venn explica o fato de a lógica não ser uma parte da matemática, apesar de poder ser simbolizada algebricamente. De acordo com Venn (1894, p.x), lógica e matemática partilham uma estrutura formal que podemos representar com recurso à álgebra simbólica. Dessas semelhanças formais não se segue, contudo, que matemática e lógica identifiquem-se. Na opinião de Venn (1894, p.xxii), a álgebra da lógica e a álgebra matemática seriam estritamente subrogativas e representariam domínios de objetos precisamente diferenciáveis: enquanto a matemática seria o estudo das quantidades, a lógica seria o estudo das classes e de suas relações.

No entanto, para sustentar sua opinião de que a lógica, embora possa ser algebricamente simbolizada, não é uma parte da matemática, Venn precisa mostrar o caráter estritamente subrogativo de seu sistema simbólico. Em outras palavras, Venn deve provar que a cada elemento componente de seu sistema simbólico corresponde algum significado lógico. A argumentação de Venn nesse tópico consiste em rechaçar um conjunto de críticas de Jevons à álgebra da lógica de Boole. Ora, dada a similaridade técnica entre a álgebra da lógica de Boole e a álgebra da lógica de Venn (Grattan-Guinness 2000, p.61), essas críticas de Jevons atingem também a essa última proposta. Em Pure Logic (1864), Jevons formula quatro objeções à álgebra da lógica de Boole. Em primeiro lugar, Jevons critica o modo de simbolização da operação de disjunção nessa lógica simbólica. Segundo Jevons, é incorreto representar a operação de disjunção inclusiva por um modo exclusivo de simbolização, tal como o 
adotado por Boole e Venn: nas álgebras da lógica desses autores, caso suponhamos que duas classes "A" e "B" podem ter membros em comum, devemos representar a disjunção de "A" e "B" por " $a+(b \cdot$ não- $a$ )" (Venn 1894, p.45-6). Em segundo lugar, Jevons critica a álgebra da lógica de Boole por, supostamente, ser inconsistente com a verdade lógica "A ou A é A". De fato, na álgebra da lógica de Boole, assim como na álgebra da lógica de Venn, devido ao modo de representação da operação lógica de disjunção, " $a+a=a$ " sequer é uma fórmula bem formada. Além disso, Jevons critica a álgebra da lógica de Boole, e, consequentemente, a lógica simbólica de Venn, por reconhecer a existência da operação de subtração lógica. Jevons argumenta que a legitimidade da operação de subtração lógica depende da legitimidade do modo como a álgebra da lógica de Boole representa a operação de disjunção (Halsted 1878, p.135). Por fim, Jevons argumenta que "os símbolos $1 / 1,0 / 0,0 / 1,1 / 0$ não estabelecem por si só qualquer significado lógico" (Jevons 1864 apud Grattan-Guinness 2000, p.56. Nossa tradução). ${ }^{12}$ Ou seja, Jevons critica a álgebra da lógica de Boole, e, consequentemente, a álgebra da lógica de Venn, por reconhecer a existência da operação de divisão lógica.

Venn responde as três primeiras críticas de Jevons chamando atenção para o caráter parcialmente convencional da representação simbólica. Por um lado, é verdade que existem alguns aspectos da representação simbólica que não são convencionais: por exemplo, se o domínio de objetos representado satisfaz a propriedade formal da associatividade, a sua simbolização algébrica, se é uma representação adequada desse domínio, necessariamente satisfaz tal propriedade. Contudo existem aspectos da representação que são convencionais. Assim, por exemplo, uma representação exclusiva é tão adequada quanto uma representação inclusiva da operação de disjunção. Naturalmente, caso se convencione representar a operação de disjunção de modo exclusivo, fórmulas como " $a+a=a$ " não são bem formadas, mas isso não significa que o sistema simbólico que respeita essa convenção seja inconsistente com uma verdade lógica tal como "A ou A é A". Significa apenas que essa verdade lógica não pode ser representada por aquela fórmula (ela pode, por outro lado, ser representada, de modo consistente, por " $a+(a \cdot$ não- $a)=a$ ") (Venn 1894, p.xv). Ora, se é legítimo representar a operação de disjunção de modo exclusivo, então também não se sustenta a critica de Jevons ao reconhecimento da operação de subtração lógica. A operação de subtração lógica possui legitimidade, e mesmo o fato de que possamos construir uma lógica simbólica com ausência dessa operação não é razão suficiente para deslegitimá-la (Venn 1894, p.50).

Ao ser questionado sobre a legitimidade da operação lógica de divisão, Boole concede que o símbolo algébrico de divisão não possui significado lógico. Apesar disso, Boole considera que esse símbolo constitui-se em elemento ideal de seu sistema simbólico, eliminável sem perda de resultados sobre o domínio representado (Van Evra 2000, p.90). A resposta de Venn é totalmente distinta da resposta de Bo- 
ole. Venn não apenas considera legítimo o uso lógico do símbolo de divisão, como atribui a esse símbolo significado lógico. Na álgebra da lógica de Venn, a aplicação da operação de divisão lógica gera como resultado uma classe de extensão indefinida (Venn 1894, p.77-8). Em especial, dado que, na álgebra da lógica de Venn, a seguinte fórmula expressa uma verdade lógica, " $a / b=(a \cdot b)+0 / 0$ (não- $a \cdot$ não- $b$ )", "0/0" significa uma classe de extensão indefinida. No entanto, tal indefinição não é atual, mas antes de caráter epistemológico: com a operação de divisão lógica representamos o desconhecimento ou irrelevância da real extensão de uma classe. Venn (1894, p.110) mostra a importância de considerar essa operação na formalização lógica recorrendo à análise dos diagramas de Venn, contraparte visual de sua álgebra da lógica. Venn apresenta seu sistema diagramático como um substituto dos diagramas de Euler. A grande vantagem dos diagramas de Venn frente aos diagramas de Euler estaria no fato de representar com um único diagrama porções parciais de informação, o que não é possível pelo sistema diagramático rival. Consideremos por exemplo que, com diagramas de Euler, não é possível representar com um único diagrama proposições categóricas universais afirmativas, como podemos visualizar na Figura 2 (Bernhard 2006, p.50-51):
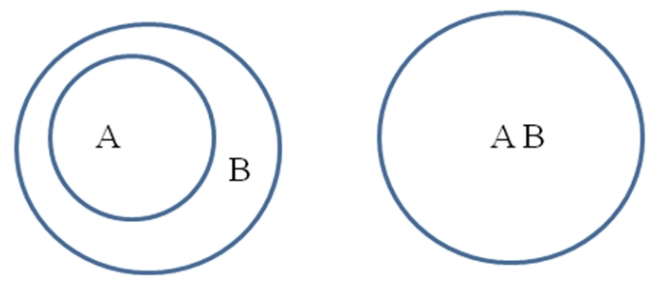

Figura 2

Podemos, por outro lado, representar proposições categóricas universais afirmativas através de um único diagrama de Venn porque, com esse sistema diagramático, não apenas o que é afirmado na proposição pode ser representado (no caso, que "não existem As que não são Bs"), mas também o que é omitido. Essa porção de informação omitida pela proposição é representada nos diagramas de Venn através da ausência de marcas em áreas específicas. À informação omitida da proposição corresponde certa extensão indefinida das classes envolvidas, seja por que essa extensão é desconhecida, seja porque é irrelevante. De todo modo, como podemos verificar na comparação entre diagramas de Euler e diagramas de Venn, considerar as porções epistemologicamente indefinidas da informação proposicional é fundamental para uma boa formalização lógica. 


\section{Conclusão}

Sumarizamos abaixo, portanto, os principais resultados alcançados nesse trabalho:

- Através de reconstrução histórica da álgebra da lógica de Venn, indicamos suas especificidades frente a outros projetos de álgebra da lógica do século XIX. Indicamos, por exemplo, que a álgebra da lógica de Venn atribui valor lógico ao reconhecimento de classes epistemologicamente indefinidas, ao contrário do que é feito na álgebra da lógica de Boole;

- Com base na sistematização de Esquisabel (2012) das funções do conhecimento simbólico, mostramos que a função ectética do conhecimento simbólico cumpre papel no processo de abstração da silogística levado a cabo na álgebra da lógica de Venn;

- Em seguida, mostramos que cumpre papel na explicação de Venn da diferença entre lógica e matemática a distinção entre dois modos em que o simbolismo algébrico pode satisfazer a função subrogativa.

Concluímos que esses resultados permitem jogar luz sobre o papel que o conhecimento simbólico cumpre no desenvolvimento das ciências formais. Assim verificamos que o processo de superação da silogística, característica marcante da lógica do século XIX, depende em Venn do desempenho da função ectética do conhecimento simbólico. Verificamos também que a análise de Venn da relação entre lógica e matemática depende da articulação das diferentes maneiras em que o conhecimento simbólico pode cumprir a função subrogativa. Ao permitirem jogar luz sobre o desenvolvimento das ciências formais, em especial da lógica, os resultados aqui alcançados oferecem modesta colaboração à discussão recente em historiografia da lógica. Sobre a historiografia da lógica simbólica e suas origens no século XIX, é incontornável a tese de van Heijenoort (1967), segundo a qual a lógica simbólica é resultado de dois esforços do século XIX, a saber, o esforço dos algebristas da lógica (autores tais como Boole, Schroeder, Venn etc) e o esforço dos lógicos matemáticos (autores tais como Peano, Frege etc). Segundo van Heijenoort, podemos formular a diferença entre esses projetos de lógica simbólica em termos de dois projetos leibnizianos: enquanto os algebristas da lógica procuraram desenvolver um calculus ratiocinator, os lógicos matemáticos procuraram desenvolver uma lingua characterica (Van Heijennoort 1967, p.324). Em sentido Leibniziano, lingua characterica ${ }^{13}$ significa um meio de representação universal, capaz de representar o domínio total de objetos nos seus elementos mais simples. Calculus ratiocinator, por outro lado, significa um cálculo a ser aplicado à lingua characterica que permite obter toda verdade sobre o domínio universal de objetos (Peckhaus 2004, p.7-8). Segundo van Heijenoort (1967, p.3246), tal diferença entre os projetos de lógica simbólica corresponderia a diferenças 
entre as lógicas representadas por seus sistemas simbólicos. A lógica simbólica seria, assim, o resultado misto da articulação de ambos os projetos do século XIX.

A tese de van Heijenoort vem sofrendo forte revisão na literatura filosófica recente. Assim, Peckhaus (2004) aponta que os critérios elencados por van Heijenoort a fim de diferenciar álgebra da lógica de lógica matemática são insuficientes. Peckhaus (2004, p.10) mostra que algebristas da lógica tais como Schroeder também caracterizaram seus sistemas lógicos nos termos de Frege, i.e., como desenvolvimentos do projeto de uma characteristica universalis. Ademais, uma teoria lógica tão sofisticada quanto a de Frege pode ser encontrada no trabalho de Schroeder (Peckhaus 2004, p.11-2). Logo, mesmo que possamos distinguir duas tradições originárias de lógica simbólica no século XIX, a raiz dessa distinção não está nos conteúdos lógicos expressos pelos diferentes sistemas. Mais promissora é a hipótese de Legris (2012) que chama atenção para o caráter epistemológico com que podemos distinguir álgebra da lógica de lógica matemática. Assim, poderíamos distinguir essas tradições chamando atenção para as diferentes funções que o conhecimento simbólico cumpre em seus sistemas lógicos (Lassalle Casanave, 2012b, p.x). De sua parte, Legris (2012) procura mostrar que no projeto lógico fregeano cumprem papel certas funções do conhecimento simbólico que ou não encontram lugar ou aparecem apenas de modo secundário na álgebra da lógica de Boole e Schroeder. Em especial sobre a tradição da álgebra da lógica, Legris (2006, p.100) verifica a passagem de um estado inicial de interesse pragmático na construção de sistemas inferenciais para um interesse semântico na compreensão das estruturas formais subjacentes à lógica simbólica. Os resultados aqui alcançados sugerem que Venn encontra-se num estado intermédio desse processo. Por um lado, os interesses de Venn no desenvolvimento da lógica simbólica são claramente pragmáticos. A superação da silogística significa em Venn o desenvolvimento de sistemas inferenciais mais potentes, o que é possível graças à eliminação de tópicos tradicionais de pesquisa lógica. Contudo em Venn também há preocupação com questões semânticas, na medida em que ele sustenta o caráter estritamente subrogativo de seu sistema simbólico, assim como uma preocupação por diferenciar precisamente a interpretação lógica da interpretação matemática do simbolismo algébrico. ${ }^{14}$

\section{References}

Angelelli, I. 1980. Traditional vs. Modern Logic: Predication Theory. Crítica: Revista Hispanoamericana de Filosofía 12(34): 103-06.

Bernhard, P. 2006. Euler Diagrams as a Visual Method of Proof in Syllogistic. Representaciones 2(2): 47-60.

Church, A. 1972. Symbolic Logic. By John Venn. The Journal of Symbolic Logic 37(3): 614-5. Durand-Richard, M-J. 2000. Logic Versus Algebra: English Debates and Boole's Mediation. 
In: J. Gasser (ed.), A Boole Anthology. Netherlands: Kluwer Academic Publishers, p.13966.

Esquisabel, O. M. 2012. Representing and Abstracting: an Analysis of Leibniz's Concept of Symbolic Knowledge. In: A. Lassalle Casanave (ed.) Symbolic Knowledge from Leibniz to Husserl. Londres: College Publications, p.1-49.

Geach, P. 1980. History of the Corruptions of Logic. In: P. Geach Logic Matters. Berkeley: University of California Press, p.44-61.

Grattan-Guinnes, I. 2000. The Search for Mathematical Roots, 1870-1940. Princeton: Princeton University Press.

Halsted, G. B. 1878. Prof. Jevons's Criticism of Boole's Logical System. Mind 3(9): 134-37.

Hansson, S. O. 2000. Formalization in Philosophy. The Bulletin of Symbolic Logic 6(2): 16275.

Kneale, W. 1948. Boole and the Revival of Logic. Mind 57(226): 149-75.

Lassalle Casanave, A. 2012a. Kantian Avatars of Symbolic Knowledge: The Role of Symbolic Manipulation in Kant's Philosophy of Mathematics. In: A. Lassalle Casanave (ed.) Symbolic Knowledge from Leibniz to Husserl. Londres: College Publications, p.51-77.

_ 2012b. Preface. In: A. Lassalle Casanave (ed.) Symbolic Knowledge from Leibniz to Husserl. Londres: College Publications.

Legris, J. 2006. Representación y Cálculo en el Ágebra de la Lógica del siglo XIX. In: Representaciones 2(2): 89-103.

- 2012. Between Calculus and Semantic Analysis: Symbolic Knowledge in the Origins of Mathematical Logic. In: A. LassalleCasanave (ed.) Symbolic Knowledge from Leibniz to Husserl. Londres: College Publications, p.79-113.

Marques, A. 2006. Identidade dos Indiscerníveis. In: J. Branquinho; D. Murcho; N. G. Gomes (eds.) Enciclopédia de Termos Lógico-Filosóficos. São Paulo: Martins Fontes, p.391-2.

Martin, W. M. 2010. Fichte's Logical Legacy: Thetic Judgement from the Wissenchaftslehre to Brentano. In: V. Waibel et al (eds.) Fichte and The Phenomenological Tradition. Göttingen: De Gruyter, p.379-405.

Peckhaus, V. 2004. Calculus Ratiocinator vs. Characteristica Universalis? The Two Traditions in Logic Revisited. History and Philosophy of Logic 25: 3-14.

Pycior, H. M. 1981. George Peacock and the British Origins of Symbolical Algebra. Historia Mathematica 8: 23-45.

Sautter, F. T. 2010. A Essência do Silogismo: Uma Abordagem Visual. In: Cognitio 11: 316-32. Schultz, S. R. 2010. Diagramas e Visualização. Manuscrito 33: 445-65.

Smith, R. 1989. Introduction. In: Aristoteles. Prior Analytics. Indianapolis: Hackett.

Van Evra, J. 2000. A Reassessment of George Boole's Theory of Logic. In: J. Gasser (ed.) A Boole Anthology. Netherlands: Kluwer Academic Publishers, p.87-99.

Van Heijenoort, J. 1967. Logic as Calculus and Logic as Language. Synthese 17: 324-30.

Venn, J. 1881. Symbolic Logic. 1a ed. London: Macmillian.

1894. Symbolic Logic. 2a ed. London: Macmillian,

Bruno Ramos Mendonça

Universidade Federal de Santa Maria

Programa de Pós-Graduação em Filosofia, Prédio 74-A, sala 2308

Campus, Bairro Camobi

Principia 16(3): 471-488 (2012). 
97105-900 Santa Maria, RS

BRASIL

bruno.ramos.mendonca@gmail.com

Resumo. Este artigo reconstroi a lógica algébrica de Venn e identifica algumas das noções filosóficas sobre a natureza do conhecimento simbólico subjacentes ao seu trabalho. Mostramos que Venn, ao enfrentar problemas filosóficos associados à sua lógica algébrica, precisa articular as noções de função ectética e de função subrogativa do conhecimento simbólico. $\mathrm{O}$ artigo esclarece essas noções a partir da sistematização das funções do conhecimento simbólico presente na literatura filosófica recente. Além disso, este artigo situa a obra de Venn entre os esforços de simbolização da lógica do século XIX, e, a partir da análise de um caso histórico, clarifica as noções de função ectética e de função subrogativa.

Palavras-chave: Conhecimento simbólico; álgebra da lógica; silogística; diagramas de Venn.

\section{Notas}

${ }^{1}$ Para uma exposição da teoria silogística, cf. Smith 1989.

2 Venn (1894, p.434-5), contrariamente a Boole, não procura fazer depender a interpretação proposicional de sua álgebra da lógica da interpretação predicativa. Nesse sentido não se encontra em Venn uma proposta como a de Boole de conceber as proposições como classes temporais - essa proposta booleana é inclusive descartada explicitamente por Venn.

3 "Identity in respect of some one characteristic only."

${ }^{4}$ Cf. Marques (2006, p. 391-2) sobre a distinção entre identidade qualitativa e identidade numérica.

5 "Take the given equations and analyse them into all their constituent elements, that is, into all the ultimate denials which they involve and which collectively make up their significance. Then take the given function, of which we are told to find the value, and make the requisite synthesis. That is, build up successively each part of it, employing for this purpose the above mentioned denials. This latter stage is really one of rejection, for we begin by developing the required function into its full complement of potential classes, and then strike out as many of these as are shown to vanish in consequence of the previous analysis. Having thus gone through the Analysis and the Synthesis there remains the third step, namely that of Elimination. It may be required to express the desired function in terms of part only of the terms involved in the equations".

${ }^{6}$ Cf. Schultz 2010 para uma exposição crítica do tema.

${ }^{7}$ Cf. Hansson 2000 para mais detalhes sobre a noção de "idealização" tal como é satisfeita pela formalização lógica.

8 "Common Logic should in fact be no more regarded as superseded by the generalizations of the Symbolic System than is Euclid by those of Analytical Geometry. [...] The narrower system has its peculiar advantage, owing to the fact that, being by comparison more concrete, it is easier for a beginner to understand; that there is thus less danger of its failing to exercise the thinking faculty and merely leading to dexterity in the use of a formula; and that it is much more closely connected with the practical experiences and needs of ordinary life. The more general system, on the other hand, has vastly extended capacity, practises much more

Principia 16(3): 471-488 (2012). 
thoroughly the faculty of abstraction, and corrects and enlarges the scientific bases of the narrower system".

${ }^{9}$ Cf. Martin 2010 para uma exposição apurada do projeto lógico de Brentano.

${ }^{10}$ Nesse sentido, pode-se argumentar que o desenvolvimento da lógica de termos levado a cabo na álgebra da lógica de Venn inclusive já está sugerido na silogística aristotélica. Para uma argumentação célebre nesse sentido, cf. Geach 1980.

11 "Whatever form is Algebraically equivalent to another, when expressed in general symbols, must be true, whatever those symbols denote.

Conversely, if we discover an equivalent form in Arithmetical Algebra or any other subordinate science, when the symbols are general in form though specific in their nature, the same must be an equivalent form, when the symbols are general in their nature as well as in their form."

12 "The symbols $1 / 1,0 / 0,0 / 1,1 / 0$, establish for themselves no logical meaning."

${ }^{13}$ Segundo Peckhaus (2004, p.5, n.5), Leibniz nunca usa a expressão "língua characterica", mas sim "lingua generalis", "lingua universalis", "lingua rationalis", "lingua philosophica", "characteristica" e "characteristica universalis". Frege e consequentemente van Heijenoort tomaram a expressão "lingua characterica" de Trendelenburg.

${ }^{14}$ Esse trabalho é resultado de pesquisa de Mestrado executada entre os períodos de Março de 2012 a Março de 2013, no Programa de Pós-Graduação em Filosofia da Universidade Federal de Santa Maria (PPGF-UFSM), sob orientação do prof. Dr. Frank Thomas Sautter. Agradeço a CAPES pelo financiamento de bolsa de mestrado, e de bolsa de missão de estudos no exterior pelo projeto CAFP "Conhecimento Simbólico e Conhecimento Gráfico", sem o que essa pesquisa não seria possível.

Principia 16(3): 471-488 (2012). 